\title{
Adaptable Design for Design Reuse
}

\author{
D. Fletcher, P. Gu \\ Department of Mechanical and Manufacturing Engineering \\ The University of Calgary, Calgary, Alberta, Canada \\ cdfletch@ucalgary.ca,gu@enme.ucalgary.ca
}

\begin{abstract}
Due to the complexity, associated cost, and general uncertainty of performing the creative design process, significant value exists in reusing the design information developed in previous design efforts. This paper outlines the current state of the art in design reuse in various fields as well as identifying both the current and future research areas. In addition, this paper describes the link between adaptable design and design reuse. Finally, as the first step in our research, this paper describes the direction that the research will take: an analysis of the use of adaptable design to enhance the design reuse process.
\end{abstract}

\section{Introduction}

Increasing competition for better product (and systems) functionality, quality, features, customization, environmental friendliness, lower cost and shorter delivery time presents unprecedented challenges for product manufacturing enterprises. These challenges cannot be completely addressed by advanced manufacturing technologies alone as some of which are originated from product design. Advanced design technologies and tools for early product design processes are critically needed where most important decisions are made with respect to the product functionality, quality, manufacturability, cost and environmental performance. This paper discusses design reuse to enhance design and design process.

Design reuse is aimed at maximizing the value of design efforts by reusing successful past design information in whole or in part for future designs. [1] From the design problem definition through to the solution, the design process requires specialized domain type knowledge and design experience which is represented as design heuristics. Based on the availability and use of the domain knowledge and heuristics, the design process can be divided into three major classifications: creative design, variant design and routine design. [2]

Creative design implies that the minimum amount of past information is used in the design process. The design process is novel; not reusing any information generated in past design activities. Variant design is a process by which an existing design is modified to meet a new set of requirements. Variant design contains elements of creative design and design reuse, in a balanced proportion. A large amount of previous design information is reused, and modified for the required functionality. Routine design is a process by which very small adjustments to an existing design are made. The process uses almost exclusively past design information with very little creative design or modification required.

It is estimated that up to $90 \%$ of all design activity is based on the variant design of existing systems. This means that more often than not when a new design problem arises, it is solved through the modification of an existing design rather than performing the design process from scratch. Due to the complexity, associated cost, and general uncertainty of performing the creative design process, significant value exists in reusing design information. [2]

The ultimate aim of design reuse is to assist the designer in the development of high quality products that meet all customer requirements while expending the least amount of effort and resources. Design reuse as a concept was developed and researched extensively in the computer science and software development fields. [1] 


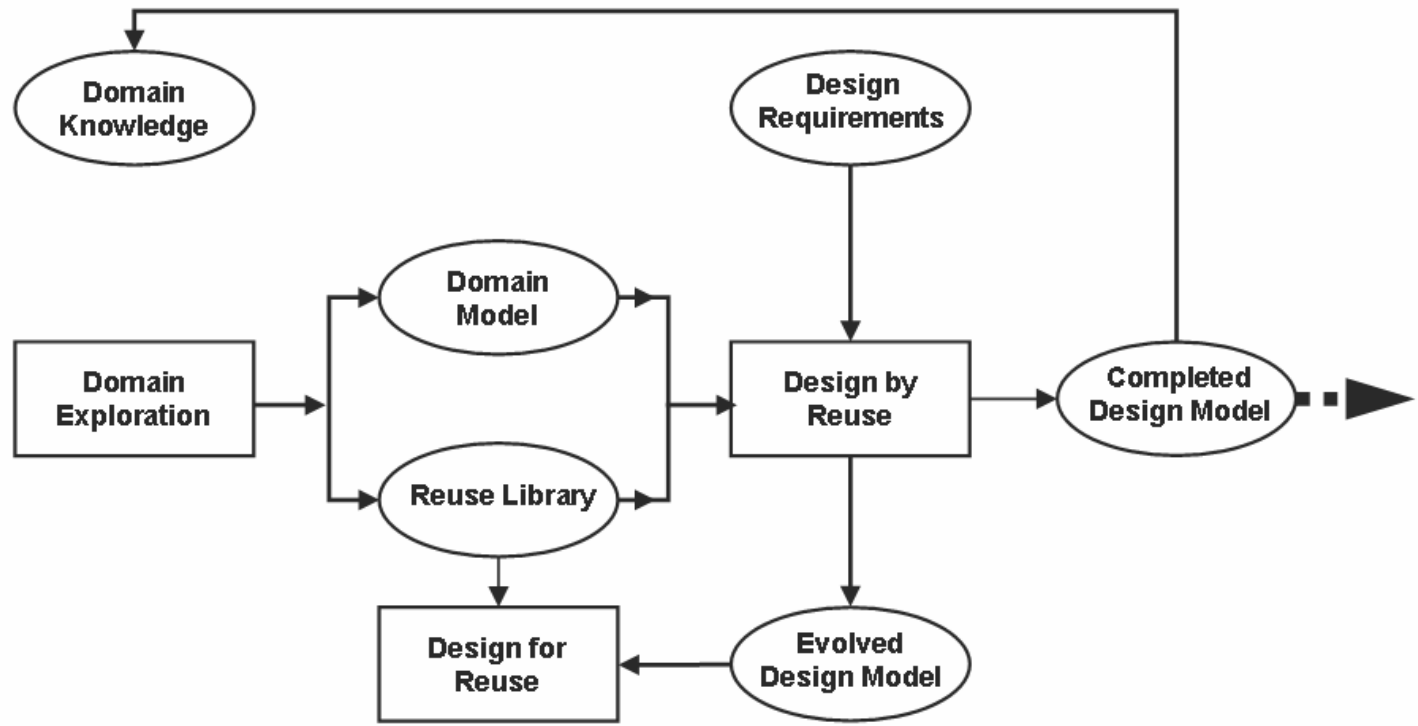

Figure 1. A design reuse system [1]

\section{Design reuse}

Through the design process, designers typically seek two types of information to reuse: design knowledge specific knowledge to answer clear questions, and design rationale - knowledge that includes patterns in the artefact information, subsystems and their relation to the entire system, as well as the analysis models associated with the generation of the previous designs. [1]

As a result of the work done in the computer science and software development fields, a significant amount of effort has been expended for the utilization of computers in the design process. The role of the computer in design reuse is significant in the following areas: Indexing of design information and information retrieval, design knowledge utilization, and design knowledge exploration and adaptation. [1] $\mathrm{CAD} / \mathrm{CAM} / \mathrm{CAE}$ knowledge databases are vital to the designer as they are used to search

through the vast amounts of legacy data and retrieve the relevant components for integration to the new design process. [3]

There has also been some research into the design process using the concepts of design reuse. Duffy et all propose the design reuse model shown in figure 1. [1] In the model, legacy information is accessed through reuse libraries to aid in the design process. Through the design process, the reuse libraries are continually updated to contain any new information generated for use at a later date.
However, even if all previously generated design knowledge and information as well as the required information management and retrial systems are in place, some designs are inherently more reusable than others. Therefore, in addition to design knowledge and information systems, our work will also explore the way to structure the design process so as to develop more reusable designs.

\subsection{Design knowledge}

Design knowledge is the sum of the information obtained throughout product life cycle such as product design specifications, product implementation instructions, manufacturing processes, product performance data, service instructions and end of life characteristics. It is noted that this is by no means an exhaustive list, just representative examples of design knowledge information.

CAD models are an integral part of design knowledge capture and retrieval. With the widespread use of CAD tools in the design process, the digital information generated is the dominant form of the design knowledge that results from the design process. Therefore, the associated CAD knowledge databases are vital to the designer to search through the vast amounts of legacy data and retrieve relevant components for integration in the new design. [3] 


\subsection{Design rationale}

Lee (1997) in [4] defines design rationale as the following:

\author{
"Design rationales include not only \\ the reasons behind a design decision \\ but also the justification for it, the \\ other alternatives considered, the \\ tradeoffs evaluated, and the \\ argumentation that led to the \\ decision"$$
\text { [Lee (1997) in [4]] }
$$

Design rationale consists of the less formalized information generated through the design process. Design rationale is the closest form to capturing the knowledge of a designer that is currently employed. Due to its informal nature and the volume of data generated, design rationale is difficult to capture, and even more difficult to disseminate in any meaningful manner. Due to the difficulties associated with design rationale, it is vary rarely recorded for reuse if at all. [4]

Design rationale as the basis behind the realization of a design and a detailed description of the design process undertaken has many potentially important uses: design verification, evaluation, reuse, teaching, communication, assistance, and documentation.

\subsection{Adaptable design}

Adaptable design is a promising methodology to increase the potential for the reuse of design information. The underlying philosophy of adaptable design is the ability to adapt an existing design to new design requirements and therefore reuse a product and the design when the requirements change.

Two distinct adaptabilities are identified: specific adaptability and general adaptability. Specific adaptability means that the adaptation requirements are known in design stage. For example, multiple product models can be replaced by a single (or smaller number) adaptable product with different variations or add-on attachments. [5] General adaptability of a product design means that such a design is generally more adaptable to accomplish adaptation requirements than otherwise.

Adaptable design consists of several key elements that distinguish adaptable design from traditional design. The first key element in adaptable design is to extend the utility of product in design (design knowledge and information) and physical form (upgrading, reuse, remanufacturing and recycling). In adaptable design, the most important information about a product is the functions which it performs. The functional requirements are realized by the physical structure of the product.

Another key element of adaptable design is the quantification of a product's adaptability so that it can be considered and compared to comparable solutions during the decision making process. A third key element of $\mathrm{AD}$ is a model of the design process used to create an adaptable product. Typically, a functionbased design process model is used to model the design process and the resulting products. [5] Therefore, adaptable design is design paradigm that creates a design environment that emphasizes design reuse.

\section{State of the art}

\subsection{Software engineering}

Design evolution and maintenance or variant design as opposed to creative design is the dominant design methodology in software development situations. [6] Also, due to the very large number of standardized electrical components used in the development of computer systems, it follows that design reuse grew from the software development and computer science fields. [1]

As a result of work done in the fields of computer science and software development, a significant amount of work has been completed in utilizing computers for the following roles: indexing and retrieval of design information, design knowledge utilization, and design knowledge exploration and adaptation. [1] The fields of software engineering and computer science represent the major users of highlevel design reuse, reusing entire components in different configurations with other components to generate new products.

\subsection{Mechanical engineering}

Design reuse of mechanical systems has been of limited to the use of common platforms, modules and key components for the development of variations of an existing product family or partial reuse of the past design in a non-systematic manner such as the cut and paste of old designs to create a new design. Due in part to increased interfacing problems within mechanical systems, design reuse is less mature in a formalized manner in mechanical design. 
Table 1. Available design reuse tools [4]

\begin{tabular}{|l|l|l|}
\hline \multicolumn{1}{|c|}{ Tool Name } & \multicolumn{1}{c|}{ Domain Type } & \multicolumn{1}{c|}{ Tool Creators } \\
\hline Design History Tool & Mechanical Design & Chen et. al, 1990 \\
\hline $\begin{array}{l}\text { An Intelligent Design Evolution } \\
\text { Management System }\end{array}$ & General Design & Thompson \& Lu, 1990 \\
\hline Process Technology Transfer Tool & General Design & Brown \& Bansal, 1991 \\
\hline Design Rationale Capture System & Manufacturing & Klein, 1992 \\
\hline Active Design Documents & HVAC Design & Garcia et. al, 1993 \\
\hline Reconstructive Derivational Analogy & Electrical Circuit Design & Britt \& Glagowski, 1995 \\
\hline gIBIS and itIBIS & Generic Design & $\begin{array}{l}\text { Conklin \& Burgis- } \\
\text { Yakemovic, 1995 }\end{array}$ \\
\hline JANUS & Kitchen Design & Fischer et. al, 1995 \\
\hline DRIM/SHARED-DRIM & Civil Engineering & Pena-Mora et. al, 1995 \\
\hline Hyper-Object Substrate & Generic Design & Shipman \& McCall, 1996 \\
\hline PHIDAS & Graphic Design & Shipman \& McCall, 1996 \\
\hline M-LAP & Generic Design & Brandish et. al, 1996 \\
\hline Device Modelling Environment & Electro-Mechanical Design & Gruber, 1990 \\
\hline SIBYL & Any Design & Lee, 1990 \\
\hline
\end{tabular}

The ideas generated in the software engineering and computer science fields are gaining greater attention for use in mechanical design. There is much active research in this field, in the areas of design knowledge and design rationale capture, as well as the dissemination of the gathered information to the designer.

\subsection{Design reuse tools}

Many software tools have been developed to aid the designer in design reuse, a few of which are summarized in Table 1.. The tools listed show a representative sample of the design rationale capture tools that have been developed along with the associated application scenario for which they were developed.

\section{Barriers to design reuse}

Design reuse, although an attractive design methodology that has the potential for significant cost savings through streamlining the design process, can be a difficult methodology to implement. Design organizations typically have difficulty in reusing design information for many reasons, including the fact that an individual designer often finds it difficult to reuse the designs, experience and insight of other engineers. [7]

Design reuse in actual practice can be of limited success due to a number of related factors. Many of the factors will often contribute in at least some respect to the failure of design reuse. [8]

Indexing and retrieval problems - designers can have difficulties in identifying relevant designs for reuse, especially as the search for information recedes further back in time and the amount of information available increases.

Misunderstanding - even when the relevant previous designs are retrieved, designers can have problems in understanding these prior designs and in applying them to the current design problems.

Modification issues - rapidly changing requirements in technology can also make previous designs difficult to modify or reuse directly. Alternatively, as more advanced technologies become available, design reuse can become difficult as the benefits of the new technology will not be realized if it is not incorporated into the design. The design rationale however is often the most valuable resource for design reuse. Designers can also underestimate the adaptation activity required to reuse and modify existing designs, and that they can attempt to modify prior designs without a clear appreciation of the reasoning underlying the design.

Satisfying tendencies - the reuse of existing solutions can lead to design inefficiency as the innovation present in the previous designs may restrict the range of options explored by the designer. Designers can become fixated on single solutions rather than exploring alternatives in order to improve choices. 
Organizational matters - design companies tend to not provide any incentives for designers to engage in design reuse, or to make their designs available for future reuse. The individual project-based project management methods in use today hamper design reuse in that generating design information in such a way that it is reusable for future efforts does not directly add value to the project at hand. The designer therefore has pressure from his superiors to not spend time on an activity that does not add value to the project at hand. Also, individual designers may be reluctant to reuse others' designs. [8]

If the process of the specification of design information for reuse is too difficult, then only few parts will be added to the design library allowing only a limited amount of information to be available for reuse at a future date. It has been shown that if the time required to reuse a part is greater than about $30 \%$ of the time required to design the part from scratch, design reuse will fail. This comparison includes the time required to locate the relevant information, read the accompanying documentation, determine and select the applicable element, modify it as required, and integrate it into the design. [9]

Successful knowledge reuse requires a solution that addresses many different areas. It is not simply a matter of developing a reuse technology for the design engineers. Special attention to incentives for contributing to and using the design repositories and the role of the intermediaries required to develop and maintain these knowledge repositories to facilitate knowledge reuse. [10] All of the barriers listed above must be addressed in order to create a process within an organization that encourages design reuse.

It should also be noted that excessive design reuse can also hurt the design process within an organization. The overuse of design reuse can lead to design fixation, and kill the creativity needed in certain aspects of the design process. [1] Creative design is an integral part of the design process used to generate alternative designs. Alternative designs outside the realm of design reuse may provide more highly optimized solutions to the design problem at hand.

\section{Future work}

\subsection{Design reuse}

The fundamental problem for design reuse is that there is no computational model of design that is tailored to support the design reuse process. Computational models need to be developed that have the ability to capture, store and disseminate the information generated through the design process: design knowledge and design rationale. [2] For the computational aspects of design reuse, three main problem areas are identified. The areas of major difficulty lie in: design information indexing and retrieval, design knowledge utilization, and the exploring and adapting of design information. [1]

Design knowledge, the characterization of the completed designs at the end of the design process has several areas that are under investigation. Several of the issues are listed below: [4]

Relationship between design rationale capture and use and the design phase

Relationship between design rationale capture and use and the domain type

Design rationale capture issues

- Difficulties in classifying information

- Difficulties in getting engineers to record rationale info

Rationale use issues

- How to best disseminate the correct information to the design engineer

Relationship to other areas

Multiple designer support

For design reuse in general, there are many issues that need to be solved. Better tools for design data management and project tracking are needed to measure the real progress towards the project milestones. As a huge amount of design information is generated during the design process, better design library management techniques are needed to bring down the required search time for relevant design information.

Documentation and support of the reusable elements of previous designs are also crucial. The documentation and support must disclose how to use a part properly without necessarily providing all of the underlying design information which can make the exercise too tedious. Another challenge is the adoption of standards that will allow the reuse of design information with appropriate consideration for the IP involved. By 
ensuring an equitable use of information, designers and organizations will be more likely to share information more freely. [9]

\subsection{Our future work}

Our work in this area focuses on how employing the adaptable design paradigms can be used to foster the design reuse process. Specifically, how can the adaptable design methodology be used to its highest potential as a design process.

The work will also explore the relationship between adaptable design and design reuse in order to better understand how to reuse previous design efforts to improve the quality and adaptability of the products generated while simultaneously shortening the design process. The resulting design framework will assist the design organization in providing higher quality products while expending the minimum amount of efforts on the design process itself.

\section{Conclusions}

Design reuse is aimed at maximizing the value of these efforts by reusing successful past design information in whole or in part for future designs. [1] From the design problem definition through to the solution, the design process requires specialized domain type knowledge and design experience - heuristics. [2] More often than not when a new design problem arises, it is solved through the modification of an existing design rather than performing the design process from scratch. Due to the complexity, associated cost, and general uncertainty of performing the creative design process, significant value exists in reusing design information. [2]

The ultimate aim of design reuse is to assist the designer in the development of high quality products that meet all customer requirements while expending the least amount of effort and resources. Design reuse as a concept was developed and researched extensively in the computer science and software development fields. [1]

In engineering design, reuse has mainly been treated as a purely technological problem, a problem that can be solved with the development and application of various computer applications. [7] Successful knowledge transfer or reuse requires a solution that addresses many different areas. It is not simply a matter of developing a reuse technology for the design engineers. Special attention to incentives for contributing to and using the design repositories and the role of the intermediaries required to develop and maintain these knowledge repositories to facilitate knowledge reuse. [10]

In addition to the potential design cost savings offered by design reuse, other significant changes in the design field will emerge. The performance of the finished product can be improved by having relevant experts design key blocks and giving all designers access to these high quality implementations of design, and reusing these blocks in future designs. As design reuse increases, the level of utilization and importance of digital media will also increase substantially as this will become the primary means of the communication and reuse of ideas.

By reusing design information, productivity in the design process can be increased as designers can reuse large portions of the design common to the new activity. Predictability in the design process is also improved as the timing and other performance characteristics of the design are better known at the outset of the project, eliminating any unexpected setbacks. [9]

\section{References}

[1] Sivaloganathan, S., Shahin, T., "Design Reuse: An Overview", Proceedings of the Institute of Mechanical Engineers, Vol. 213 Part B, 1999.

[2] Gao, Y., Zeid, I., Bardasz, T., "Characteristics of an Effective Design Plan System to Support Reuse in Case-Based Mechanical Design", Knowledge Based Systems, Vol. 10 No. 6, 1997

[3] Regli, W., Cicirello, V., "Managing Digital Libraries for Computer-Aided Design", Computer-Aided Design 32, 2000.

[4] Burge, J., Brown, D., "Design Rationale Types and Tools", AI in Design Group, Computer Science Department, The Worcester Polytechnic Institute, 1998

[5] Gu, P., Hashemian, M., Nee, A., "Adaptable Design", Annals of the CIRP, 2004

[6] Arango, G., Schoen, E., Pettengill, R., “A Process for Consolidating and Reusing Design Knowledge", IEEE, 1993. 
[7] Busby, J. S., "The Problem With Design Reuse: An investigation into outcomes and antecedents", Journal of Engineering Design, Vol. 10 No. 3, 1999

[8] Ball et. al., "Representing Design Rationale to Support Innovative Design Reuse: A Minimalist Approach", Automation in Construction 10, 2001

[9] Girczyc, E., Carlson, S., "Increasing Design Quality and Engineering Productivity Through Design Reuse", Proceedings of the $30^{\text {th }}$ ACM/IEEE Design Automation Conference, 1993

[10] Markus, M., L., "Toward a Theory of Knowledge Reuse: Types of Knowledge Reuse Situations and Factors in Reuse Success", Journal of Management Information Systems, Vol. 18, No. 1, 2001 